\title{
Microbiological surveillance in lung disease in ataxia telangiectasia
}

\author{
To the Editor:
}

Ataxia telangiectasia is a progressive, neurodegenerative disease causing immunodeficiency, an increased risk of malignancy and respiratory disease, such as chronic sinopulmonary infection, aspiration, bronchiectasis and interstitial lung disease. The leading causes of premature death in ataxia telangiectasia are cancer and respiratory disease [1]. Published recommendations about management of pulmonary problems in ataxia telangiectasia are based on extrapolation from other conditions, which highlights the gaps in the understanding of this rare disease. Little is known about airway infections in ataxia telangiectasia [2]. Previous work has described retrospective data sets from single small clinics [3] or review of medical records in a large series of ataxia telangiectasia patients with chronic respiratory disease $[4,5]$. The microbiology in these patients was found to be unlike that of any other primary immune deficiencies and had more similarities to that seen in patients with cystic fibrosis. In cystic fibrosis, it has been long recognised that the organisms infecting the lower airway will determine treatment, quality of life, prospects for transplantation and overall survival. The accurate and prompt identification of respiratory pathogens is essential for ensuring timely commencement of eradication treatment for early infection with bacterial pathogens, the use of appropriate long-term and rescue antibiotics for those with chronic bacterial infection, and the application of appropriate infection control measures [6]. Hence, microbiological surveillance is standard practice in cystic fibrosis clinics internationally. In patients with other primary immunodeficiencies, proactive treatment with immunoglobulin replacement therapy and broad-spectrum antibiotics prevents the development of bronchiectasis and alters the natural course of respiratory disease [7]. A state of the art document [2] recognises that future research in ataxia telangiectasia should address the identification of common organisms associated with sinopulmonary infections in ataxia telangiectasia and the incidence of bacterial airway colonisation in these patients.

We aimed to describe the microbiology of the respiratory tract in patients attending the UK National Paediatric Ataxia Telangiectasia Clinic (Nottingham, UK) in order to guide blind antimicrobial therapy and to inform future studies to improve the evidence base for ataxia telangiectasia treatment. Samples were collected prospectively for microbiological surveillance from consecutive patients attending at scheduled non-urgent clinic visits, usually every 2 years. For children who were able to expectorate, sputum samples were obtained. For non-expectorating children, oropharyngeal cough swabs were obtained by asking the patient to cough onto a cotton-tipped swab placed in, but not touching, the posterior pharynx, as described in cystic fibrosis [8], and were send for bacteriology. Our microbiology laboratory only reports culture results, and does not report on the presence or absence of leukocytes or epithelial cells. Viral throat swabs were send in viral transport medium and analysis was performed by in-house PCR assays for a panel of respiratory viruses (influenza $\mathrm{A}$ and $\mathrm{B}$, respiratory syncytial virus, human metapneumovirus, parainfluenza $1-3$, rhinovirus, and adenovirus). We also prospectively collected data on current cough as a verbal category descriptive score [9], which is a score assigned to a description for daytime and night-time cough. The scoring was performed as follows. Daytime score: 0 , no cough; 1 , cough for one or two short periods only; 2 , cough for more than two short periods; 3 , frequent coughing but does not interfere with school or other activities; 4, frequent coughing that interferes with school or other activities; and 5, cannot perform most usual activities due to severe coughing. Night-time score: 0 , no cough at night; 1a, cough on waking only; 1 b, cough on going to sleep only; 2 , awoken once or awoken early due to coughing; 3 , frequent waking due to coughing; 4, frequent coughs most of the night; and 5, distressing cough.

We also recorded the findings of respiratory examination, peak cough flows and lung function (in children who were able to perform spirometry), any new treatment prescribed, and current maintenance antibiotic or immune replacement therapy. We do not have reliable data on the prescription of or adherence to acute treatment antibiotics in the period before the cultures were taken.

When available, results of microbiology samples taken at the local centre were also included in the dataset (table 1). These data are incomplete and probably subject to selection bias, so accurate prevalence cannot be determined. Clinical assessment as detailed earlier, including obtaining microbiological samples, is considered standard paediatric respiratory care and, hence, this service evaluation did not require ethical approval. 


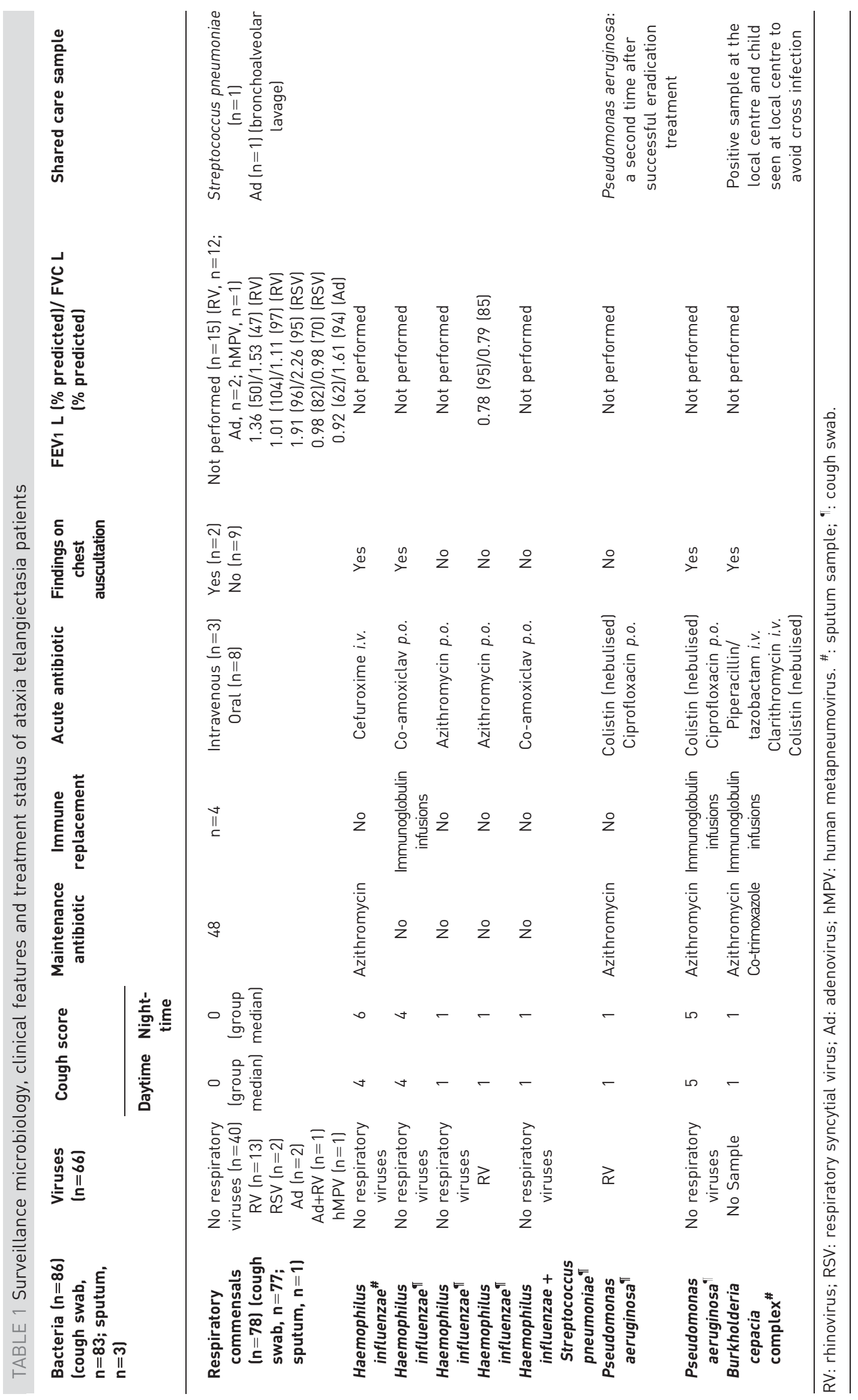


70 children were seen on 110 occasions (five children seen three times, 30 seen twice and 35 seen once) between May 2009 and May 2013. The median age was 9.22 years (range 2.32-17.10 years). We were unable to obtain a sample for bacteria on 24 visits (median age 9.3 years, 13 males and 11 females) and viruses on 44 visits because some children would not cooperate with the cough swabs (bacteria, $\mathrm{n}=21$; viruses, $\mathrm{n}=20$ ) or there was in error in sample processing (bacteria, $n=3$; viruses, $n=24$ ). Results are summarised table 1 : of the 86 samples ( 83 cough swabs and three sputum samples) (median age 9.24 years, 40 males and 46 females) obtained for bacteria, respiratory commensals were reported in 78 while eight were positive for one or more bacteria; our microbiology laboratory does not report any more details of the respiratory commensals. Two out of three sputum samples and six out of 83 cough swabs were positive for bacteria. There were too few sputum samples to allow a comparison between cough swabs and sputum samples.

Of the 66 samples obtained for viruses, no respiratory viruses were confirmed in 45 (40 in the bacterianegative group and five in the bacteria-positive group); one or more respiratory viruses were reported in 21 samples (19 in the bacteria-negative group and two in the bacteria-positive) group.

$52(74 \%)$ children were prescribed maintenance antibiotics (azithromycin, $n=42$; others, $n=10$ ). The decision to commence prophylactic antibiotics was made either at the national clinic by the immunologist or pulmonologist, or at the local referring clinic. Four out of five children who were positive for Haemophilus influenzae or Streptococcus pneumoniae were not on prophylactic antibiotics at that time and were subsequently prescribed prophylaxis. Three children who were positive for P.aeruginosa or Burkholderia cepacia complex were already on prophylactic antibiotics, reflecting the severity and advanced stage of their disease.

19 children on 20 occasions were prescribed additional antibiotics either based on symptoms, findings on examination or the finding of a positive sample for bacteria (intravenous antibiotics, $n=5$; oral antibiotics, $\mathrm{n}=12$; combination of oral and nebulised antibiotic for Pseudomonas eradication, $\mathrm{n}=2$ ). Three children with positive bacterial samples had a cough score of 4 or more while five children with positive samples had a cough score of 1 . These children were prescribed additional antibiotics despite minimal or absent symptoms based only on the positive bacterial swab (table 1). The low cough score is likely to reflect either an absence of symptoms (true surveillance microbiology and a positive sample could just reflect colonisation rather than infection), under-appreciation of the nature of the wet-sounding cough or the diminished ability to cough due to weakness. The median cough score in the negative bacterial group was 0 but 11 children were prescribed additional antibiotics based on clinical assessment by an experienced respiratory paediatrician. In such cases, indications for treatment were one or more of a wet cough or rattles, an abnormal respiratory examination, or a cough score $\geqslant 3$ (table 1). It is likely that in some of these children, the symptoms could have been related to a viral infection, although none of the children were febrile during this scheduled non-urgent clinic visit. Unfortunately, too few infected children were able to perform spirometry or cough peak flow (either because they were too young or had poor technique) to permit any correlation with infection status (table 1).

Seven $(12 \%)$ children were receiving immunoglobulin replacement therapy. Three out of eight children with samples positive for bacteria were on replacement therapy.

SCHROEDER and ZEILEN [4], in their retrospective series of 79 out of 101 ataxia telangiectasia patients with probably much more severe, chronic progressive pulmonary disease with intermittent acute exacerbations and irreversible radiographic changes, or who had pulmonary disease listed as the cause of their death, found a higher number (patients $<15$ years of age; 25 out of 27 positive for Staphylococcus aureus, H. influenzae or S.pneumoniae and 35 out of 47 older patients positive for P.aeruginosa) of positive respiratory cultures. This retrospective analysis was of children with established pulmonary disease, while our data are by far the largest prospective microbiology report in all children with ataxia telangiectasia at a routine clinic visit. We report here that even when children were attending for a routine visit, surveillance microbiology samples were positive for bacteria in 9\% (eight out of 86 samples) and for viruses in 32\% (21 out of 66 samples). Based on symptoms or the microbiology, new antibiotic treatment was prescribed in $17 \%$ (19 out of 110 consultations). As in other series [3, 4] we did not find any opportunistic infections.

SCHROEDER and ZEILEN [4] found that progression of lung disease accelerated after patients were diagnosed with bronchiectasis and argue that early aggressive therapy of infections/colonisation of airway disease is likely to improve the quality and length of life in these patients as in cystic fibrosis. Hence, microbiological surveillance is very important and we have shown that a proportion of samples are indeed positive for these ataxia telangiectasia patients.

There are limitations to this study. It is an observational, although prospective, review, the cultures were taken at long time intervals, and most patients were only sampled once, with limited data being available from shared care hospitals. Furthermore, we relied on upper airway cultures. The number of children with 
positive samples is small but does raise the important point that prospective microbiological surveillance at scheduled non-urgent clinic visits (as opposed to retrospective analysis of samples obtained in children with already established disease) does inform current and future management strategies in ataxia telangiectasia. We cannot determine the clinical relevance of these isolates without longitudinal and, preferably, interventional studies; even in conditions like cystic fibrosis, which have been much more widely studied, it is difficult to be sure of the relevance of an upper airway culture. The difference between colonisation and infection as well as the concordance between upper airway and lung (lower airway) cultures also remains under debate in all conditions studied $[10,11]$. We also only have very limited spirometric data on these children, who found spirometry difficult because of either or both of age and neurodisability.

Colonisation of the upper airway may precede spread to the lower airway in cystic fibrosis patients [12] and the upper airway may act as a reservoir for certain bacteria in patients of all ages with cystic fibrosis [13]. Detection of these microorganisms from the upper airway at an early stage, i.e. before colonisation of the lungs, might be important.

Upper airway infections are reported in a significant proportion of ataxia telangiectasia patients $[3,4,5,14]$. Hence, in ataxia telangiectasia patients, it is important to study the microbiology of respiratory infections in order to develop better management strategies because timely identification and treatment of respiratory infections may help preserve lung function in children with ataxia telangiectasia and protect them from progressive airway disease.

Viral respiratory infections are associated with substantial morbidity among the general population but respiratory viruses are of even greater significance for people with chronic respiratory diseases or immune deficiencies. Viral infections in cystic fibrosis are associated with increased respiratory morbidity and disease progression [15]. The impact of respiratory viruses in ataxia telangiectasia should therefore not be underestimated.

A high proportion of children were prescribed maintenance antibiotics, on inconsistent and non-evidence based criteria. The number of children on immunoglobulin replacement therapy was similar to published literature [5]. Both these may have affected the results, but the interventions reflect current clinical practice. Previously work from this clinic described sinopulmonary infections in ataxia telangiectasia patients but did not report microbiology [14]. We are prospectively collecting data on immune function, lung function, genetic mutations and level of ATM (ataxia telangiectasia-mutated) kinase activity, imaging as clinically indicated, and microbiology in these patients to describe any important associations of respiratory infection. However, it is well known that immune deficiency in ataxia telangiectasia is variable and can only be partially explained by the type of mutations present [14], that there is a poor correlation between laboratory indices of immune deficiency and clinical susceptibility to infections, and that progression of the immunological abnormalities over time is unusual [5]. The progression of lung disease is likely to be multifactorial, including recurrent aspiration, discoordinate and ineffective cough increasing the risk for respiratory infections and not just related to immune deficiency.

In summary, we report that positive cultures at microbiological surveillance for bacterial and viral respiratory infections at scheduled non-urgent clinic visits are relatively common in ataxia telangiectasia. Gram-negative rod-shaped bacteria are not rare. We therefore suggest that a routine microbiological surveillance should be undertaken and a low threshold for aggressive antibacterial treatment is warranted. Ataxia telangiectasia-specific, evidence-based guidelines are urgently needed, and these will require proper randomised controlled trials of treatments.

\section{0} @ERSpublications

Positive results on microbiological surveillance at scheduled non-urgent clinic visits are common in ataxia telangiectasia http://ow.ly/t71Gr

Jayesh M. Bhatt ${ }^{1}$ and Andrew Bush ${ }^{2}$

${ }^{1}$ National Paediatric Ataxia Telangiectasia Clinic, Nottingham, and ${ }^{2}$ Imperial College and Royal Brompton Harefield NHS Foundation Trust, London, UK.

Correspondence: J.M. Bhatt, Nottingham Children's Hospital, Queens Medical Centre, Derby Road, Nottingham, NG7 2UH, UK. E-mail: jayesh.bhatt@nuh.nhs.uk

Received: Aug 132013 | Accepted after revision: Jan 242014 | First published online: Feb 132014

Support statement: A. Bush was supported by the NIHR Respiratory Disease Biomedical Research Unit at the Royal Brompton and Harefield NHS Foundation Trust and Imperial College London.

Conflict of interest: None declared. 


\title{
References
}

1 Morrell D, Cromartie E, Swift M. Mortality and cancer incidence in 263 patients with ataxia-telangiectasia. J Natl Cancer Inst 1986; 77: 89-92.

2 McGrath-Morrow SA, Gower WA, Rothblum-Oviatt C, et al. Evaluation and management of pulmonary disease in ataxia-telangiectasia. Pediatr Pulmonol 2010; 45: 847-859.

3 Bott L, Lebreton J, Thumerelle C, et al. Lung disease in ataxia-telangiectasia. Acta Paediatr 2007; 96: 1021-1024.

4 Schroeder SA, Zielen S. Infections of the respiratory system in patients with ataxia telangiectasia. Pediatr Pulmonol 2014; 49: 389-399.

5 Nowak-Wegrzyn A, Crawford TO, Winkelstein JA, et al. Immunodeficiency and infections in ataxia-telangiectasia. J Pediatr 2004; 144: 505-511.

6 Cystic Fibrosis Trust Microbiology Laboratory Standards Working Group. Laboratory standards for processing microbiological samples from people with cystic fibrosis. Bromley, Cystic Fibrosis Trust, 2010.

7 Haidopoulou K, Calder A, Jones A, et al. Bronchiectasis secondary to primary immunodeficiency in children: longitudinal changes in structure and function. Pediatr Pulmonol 2009; 44: 669-675.

8 Equi AC, Pike SE, Davies J, et al. Use of cough swabs in a cystic fibrosis clinic. Arch Dis Child 2001; 85: 438-439.

9 Chang AB, Newman RG, Carlin JB, et al. Subjective scoring of cough in children: parent-completed vs childcompleted diary cards vs an objective method. Eur Respir J 1998; 11: 462-466.

10 Berkhout MC, Rijntjes E, El Bouazzaoui LH, et al. Importance of bacteriology in upper airways of patients with Cystic Fibrosis. J Cyst Fibros 2013; 12: 525-529.

11 Bonestroo HJ, de Winter-de Groot KM, van der Ent CK, et al. Upper and lower airway cultures in children with cystic fibrosis: do not neglect the upper airways. J Cyst Fibros 2010; 9: 130-134.

12 Taylor CJ, McGaw J, Howden R, et al. Bacterial reservoirs in cystic fibrosis. Arch Dis Child 1990; 65: 175-177.

13 Mainz JG, Naehrlich L, Schien M, et al. Concordant genotype of upper and lower airways $P$. aeruginosa and S. aureus isolates in cystic fibrosis. Thorax 2009; 64: 535-540.

14 Staples ER, McDermott EM, Reiman A, et al. Immunodeficiency in ataxia telangiectasia is correlated strongly with the presence of two null mutations in the ataxia telangiectasia mutated gene. Clin Exp Immunol 2008; 153: 214-220.

15 van Ewijk B, van der Zalm M, Wolfs T, et al. Prevalence and impact of respiratory viral infections in young children with cystic fibrosis: prospective cohort study. Pediatrics 2008; 122: 1171-1176.

\section{Performance of the revised Geneva score in patients with a delayed suspicion of pulmonary embolism}

\author{
To the Editor:
}

Establishing a prompt diagnosis of acute pulmonary embolism is a diagnostic challenge, as the clinical presentation ranges from haemodynamic shock to very subtle symptoms mimicking those of other cardiovascular or pulmonary diseases [1]. This diverse presentation facilitates diagnostic delay and, consequently, also a delay in treatment initiation, which might be an important prognostic indicator for patients with acute pulmonary embolism [1]. The standard diagnostic algorithm for suspected acute pulmonary embolism consists of sequential pre-test probability determination, D-dimer testing and computed tomography pulmonary angiography (CTPA) [2]. The pre-test probability can be estimated using a validated clinical decision rule (CDR), such as the Wells score and the revised Geneva score (RGS) $[3,4]$. In addition to an excellent sensitivity and specificity, the main advantage of this diagnostic algorithm is that $20-30 \%$ of all patients with a clinical suspicion can be managed without CTPA, since an unlikely clinical probability in combination with a normal high-sensitive D-dimer test result has been shown to accurately rule out acute symptomatic pulmonary embolism [5]. The significance of the appropriate use of this diagnostic management strategy in patients with suspected pulmonary embolism has been highlighted by a prospective cohort study [6]. In patients with inappropriate diagnostic management, the diagnostic failure rate was $7.7 \%$, compared to $1.2 \%$ for those patients in whom pulmonary embolism was ruled out according to the strategy $(\mathrm{p}<0.001)$. Importantly, symptoms suggestive of pulmonary embolism that could also be ascribed to underlying cardiopulmonary diseases (e.g. heart failure or chronic lung disease) were identified as an important factor for diagnostic delay. Notably, uncertainty exists upon the validity of the recommended diagnostic algorithm in patients with a delayed clinical presentation, since both the pre-test probability and the D-dimer test result may be affected by the longer presence of blood clots in the pulmonary artery tree [7]. In addition, most diagnostic landmark studies only considered patients with an acute onset of symptoms $[3,8]$. A recent study reported that a delay in clinical presentation did not influence the safety of ruling out acute pulmonary embolism in the case of both an unlikely clinical 